\title{
TUBERCULOSIS AS A POTENTIAL PITFALL ON FDG PET/CT IN PATIENTS WITH HODGKIN'S LYMPHOMA - A CASE REPORT
}

\author{
Pavel Bochev ${ }^{1}$, Anelia Klisarova ${ }^{1}$, Yana Bocheva ${ }^{2}$ \\ ${ }^{1}$ Department of Nuclear Medicine and ${ }^{2}$ Central Clinical Laboratory, \\ St. Marina University Hospital of Varna
}

\begin{abstract}
FDG-PET is an essential diagnostic tool in the management of Hodgkin's lymphoma (HD). However, a lot of benign conditions are well-known to present with high FDG uptake thus mimicking malignant activity. Tuberculosis (TBC) is an infection with known high FDG accumulation and should be suspected in patients with lymphoma and those treated with chemotherapy.

CASE REPORT: We present a 19-year old female with mediastinal Hodgkin's lymphoma who had discrete lung changes on presentation. After initial chemotherapy, patient's status worsened, with CT scan showing complete resolution of the mediastinal mass along with progression in the lung. Patient was restaged and mediastinal and lung involvement was proven. Therapy was escalated with further worsening and newlyfound liver lesions. TBC was clinically suspected although not objectively proven. FDG PET scan revealed high activity infiltrative lung changes, diffuse pleural activity, active liver lesions and celiac lymph nodes. However, changes could not be addressed as malignant, due to TBC or to both. Pleural biopsy revealed TBC. Patient received anti-TBC treatment only. The follow-up FDG PET scan revealed almost complete resolution of all the changes consistent with complete remission of the lymphoma and good treatment response of TBC.

CONCLUSION: Based on initial CT report we consider this case a coexistence of TBC and HD at initial presentation with further worsening rather than a newly-developed TBC on an immunosupressed ground. FDG PET is an excellent tool in HD management, but falsely positive results from TBC should be kept in mind, especially when lung in involved.
\end{abstract}

Key words: FDG PET/CT, Hodgkin's lymphoma, tuberculosis, diagnosis, treatment

\section{INTRODUCTION}

FDG PET/CT (positron emission tomography/ computer tomography with fluorodesoxyglucose) is an essential imaging modality in staging and

Address for correspondence:

Pavel Bochev, MD, PhD

Department of Nuclear Medicine,

St. Marina University Hospital of Varna

1 Hristo Smirnenski Str, 9010 Varna, Bulgaria

E-mail:pbochev@yahoo.com

Received: August 30, 2012

Accepted: January 15, 2013 treatment response assessment as well as a powerful prognostic tool in various types of lymphomas with Hodgkin's lymphoma (HD) being the primary indication (5). FDG accumulation represents the glucose utilization that is generally increased in malignant tumors. Unfortunately, the nature of the radiopharmaceutical - fluorinated desoxiglucose makes it quite an unspecific marker. The potential causes of high glucose/respectively FDG utilization, apart from malignant tumors are numerous $(1,7,8)$. The most common concern remains inflammation, although nonspecific inflammatory changes present with less prominent activity and in case of cautious 
interpretation their role as a potential pitfall is overrated. This cannot be directly addressed to granulomatous inflammation such as tuberculosis (TBC) and to specific granulomatous diseases such as sarcoidosis. Their activity could be very high and definitely indistinguishable from malignancy (1-3,68). Adequate FDG PET/CT reading can only be made in the context of the overall patient's data, including clinical, imaging, laboratory data, etc.

\section{CASE REPORT}

We hereby present a case of a 19-year old female with Hodgkin's lymphoma with primary mediastinal location. The patient's first visit was due to persistent dry cough. The chest $\mathrm{x}$-ray revealed mediastinal mass with lymphoma being the primary differential diagnosis. The patient was staged clinically and with conventional imaging methods. The initial staging CT (12/2009) revealed mediastinal mass as a primary finding, but noted discrete circumscribed infiltrative/ fibrotic changes in the upper lobe of the right lung. Biopsy from the mediastinal mass was obtained by video-assisted thoracoscopy (VATS) with a result of sclero-nodular form of Hodgkin's lymphoma (confirmed by immunohistochemistry, too). The patient was referred for a first-line chemotherapy with initial improvement of the symptoms and laboratory tests. After three ABVD cycles (from 01/2010), patient's status worsened with CT scan showing complete resolution of the mediastinal mass along with progression of the changes in the lung with further enlargement of the infiltrate and initial abscess formation. The patient was restaged and upstaged as being with mediastinal and lung involvement (stage IVB). Therapy was escalated to BEACOPP with further worsening and newlyfound liver lesions. The symptoms of cough, dispnea, febrility, night sweating and overall fatigue despite adequate treatment received for the initial diagnosis raised clinical suspicions of TBC, however, the results from the laboratory tests and sputum provided no proof of that. An FDG PET scan was scheduled after two cycles of BEACOPP chemotherapy. PET/CT was performed on a Phillips Gemini TF PET/CT as a standard whole body technique after initial patient preparation, overnight fasting and refrain from

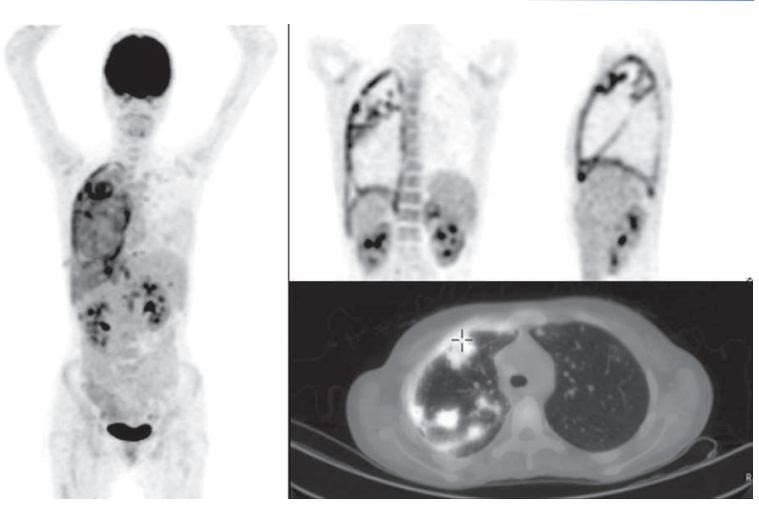

Fig. 1. Restaging FDG PET/CT reveals extremely high diffuse bilateral pleural activity as well as activity in the lungs, celiac lymph nodes and several foci of liver uptake

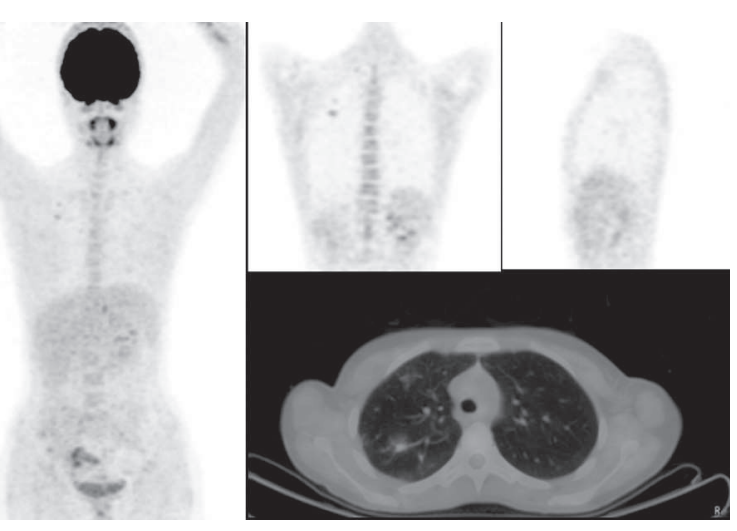

Fig. 2. Follow-up FDG PET/CT reveals almost complete normalization of the activity in the pleura, lymph nodes and liver. The lung activity is almost cleared with small foci of moderate residual uptake in the right upper lobe

tobacco and alkaloid drinks, creatinine and blood glucose testing. The patient had been administered $0,13 \mathrm{mCi} / \mathrm{kg}$ FDG and was scanned after $60 \mathrm{~min}$ uptake period. The FDG scan revealed high activity infiltrative lung changes, diffuse pleural activity, active liver lesions and celiac lymph nodes (Fig. 1). Thesefindings could be addressed either tolymphoma (node involvement, hepatic activity), or to TBC (lung activity and, especially, the pattern of diffuse pleural involvement), or to both. The conclusion, however, was equivocal, with TBC being the main concern and the pleural involvement being the major pathology requiring treatment. Chemotherapy was ceased and tuberculostatic treatment was initiated. Excision pleural biopsy revealed TBC. Patient's clinical status rapidly improved. The follow-up FDG PET scan 
(06/2010) revealed almost complete resolution of any changes consistent with complete remission of $\mathrm{HD}$ and good treatment response of TBC (Fig. 2).

\section{DISCUSSION}

In this case we emphasize the coexistence of two FDG avid conditions such as TBC and Hodgkin's lymphoma which are hardly differentiated either clinicallyor byimaging. Lung damage is not a common but possible site of involvement in lymphoma and the findings on CT could mimic other benign conditions such as inflammatory infiltrates and TBC. Thus CT diagnosis is not definite (4). Neither is FDG PET/CT due to the fact that $\mathrm{TBC}$ results in an inflammation with extremely high glucose consumption and is indistinguishable from malignancy. The ReedSternberg (RS) cells represent a small part of the lymph node pathology with lymphocyte infiltration being the major cell population in HD. Accumulation in RS cells alone can't explain the invariably high HD FDG avidity. This activity could be addressed to the lymphocyte population that is present in TBC granuloma, too. On the other hand, lymphoma as is and its chemotherapeutic treatment suppress immune response and make a room for opportunistic infections such as TBC $(3,4)$.

In this case we believe the lung TBC was preexisting (staging CT findings were not reported as suspicious for TBC but they did not exclude it, too). The fact that the abscess formation started at the site of the initial infiltrate also leads to that conclusion. During the course of the disease we detected discordant changes in the primary lymphoma mass in the mediastinum, which disappeared after three cycles of chemotherapy and the lung alterations that worsened. A misleading clue in the initial PET/ CT scan was the liver and celiac node involvement, which could be addressed to HD, but can be present in disseminated TBC, too (6). We found this case edifying in decision making based on overall patient's data, rather than differential diagnosis alone because no test in this case was able to discriminate both conditions due to their concomitant presentation.

\section{REFERENCES}

1. Bochev, P., A. Klisarova, Z. Dancheva, B. Chaushev, B. Balev. Imaging artefacts and pitfalls in 18F-FDG-PET/CT interpretation.- Rentgenol. $i$ radiol., 2011, No 1, 271-286 (in Bulgarian).

2. Chaushev, B., P. Bochev, I. Krasnaliev, A. Klisarova, Z. Georgieva, K. Yordanov, et al. The role of PET/CT for differential diagnosis of pulmonary tuberculosis and lung cancer: case report.- Scr. Sci. Med. (Varna), 44, 2012, No 1, 11-15.

3. Falagas, M. E., V. D. Kouranos, Z. Athanassa, P. Kopterides. Tuberculosis and malignancy.- Q. J. Med., 103, 2010, No 7, 461-487.

4. Hofmeyr, A., W. F. Lau, M. A. Slavin. Mycobacterium tuberculosis infection in patients with cancer, the role of 18 -fluorodeoxyglucose positron emission tomography for diagnosis and monitoring treatment response.- Tuberculosis (Edinb.), 87, 2007, No 5, 459-463.

5. NCCN Clinical practice guidelines in oncology Hodgkin lymphoma. V.2/2012. Available on: www. nccn.org

6. Nordin, A. J., C. Rossetti, N. A. Rahim. Disseminated tuberculosis infection: a 'super'18FFDG PET/CT appearance.- Eur. J. Nucl. Med. Mol. Imaging, 36, 2009, No 5, p. 882.

7. Metser, U., E. Miller, H. Lerman, E. Even-Sapir. Benign nonphysiologic lesions with increased 18FFDG uptake on PET/CT: characterization and incidence.- Am. J. Roentgenol., 189, 2007, No 5, 1203-1210.

8. Zhuang, H., J. Q. Yu, A. Alavi. Applications of fluorodeoxyglucose-PET imaging in the detection of infection and inflammation and other benign disorders.- Radiol. Clin. North Am., 43, 2005, No 1, 121-134. 